\title{
Electrodeposition of Adherent Polypyrrole Film on Titanium Surface with Enhanced Anti-corrosion Performance
}

\author{
Jilin Tan ${ }^{1}$, Zhong Zhang ${ }^{2, a}$ and Dongtao $\mathrm{Ge}^{1, \mathrm{a}}$ \\ ${ }^{1}$ Department of Biomaterials, College of Materials, Xiamen University, Xiamen 361005, China \\ ${ }^{2}$ Department of Stomatology, The Affiliated Zhongshan Hospital of Xiamen University, Xiamen 361004, China
}

\begin{abstract}
A method of producing extremely adhesive polypyrrole film on Titanium (Ti) substrate was investigated. The Ti substrate was chemically pretreated and then modified by polydopamine (PDA), the polypyrrole film synthesized by electrochemical method on the treated Ti substrate displayed good adhesion and enhanced anticorrosion performance. The study of the corrosion process was conducted through open circuit potential, tafel polarization and alternating current impedance test. The adhesive polypyrrole film coated titanium showed higher positive shift in corrosion potential and lower corrosion rate, indicating the great enhanced anti-corrosion performance.
\end{abstract}

\section{Introduction}

Polypyrrole (PPy), one of the typical conducting polymers (CPs), has been widely investigated in metal anti-corrosion applications due to its good air and water stability, high conductivity and ease of synthesis at room temperature $[1,2]$. However, the poor adhesion force between the PPy film and substrate material seriously inhibit its further application[3]. Although lots of significant progress has been made to improve its adhesion problem, there still has the desire to find a mild and effective method[4, 5].

Dopamine (DA), a biological neurotransmitter, which has a similar structure to the essential adhesive component of mussel protein, was famous for its good adhesion and easy synthesis[6, 7]. The good adhesion property and the ability to be secondary modified by other molecules make it a suitable material for improving the adhesion force between films and substrate by being used as an interlayer[8].

In the present study, we investigate an effective method to produce adherent PPy film on Ti substrate. Before electrosynthesis of PPy film, the Ti substrate was chemically pre-treated and modified by PDA interlayer. The obtained PPy film showed good adhesion force and enhanced anti-corrosion performance.

\section{Experimental Section}

Pyrrole (Py, $98 \%$ ), Sodium dodecyl benzene sulfonate (SDBS, $95 \%$ ) and DA was purchased from $\mathrm{J} \& \mathrm{~K}$ Chemical Technology. Titanium foil $(99.7 \%, 1.5 \times 2.5 \mathrm{~cm})$ was purchased from Alfa Aesar. All other chemicals were of analytical grade and were used as received.
Ti surface was chemically pretreated by dipping the Ti pieces into the solution contained $0.5 \mathrm{M} \mathrm{NaOH}$ and 1.0 $\mathrm{M} \mathrm{H}_{2} \mathrm{O}_{2}$ for 10 minutes at room temperature. Polydapomine film was deposited on Ti surface via the solution oxidation method reported by Lee et al[6]. Ti pieces were immersed into a dopamine solution $(2 \mathrm{mg} / \mathrm{ml}$ DA in $10 \mathrm{mM}$ Tris, $\mathrm{pH} 8.5$ ) for 24 hours, and then washed with ultrapure water and dried. The electrodeposition of PPy film was performed from an electrolyte solution containing $0.2 \mathrm{M}$ SDBS and $0.2 \mathrm{M}$ Py monomer via three electrode system. Platinum wire electrode, saturated calomel electrode (SCE), and $\mathrm{Ti}$ electrode was used as counter electrode, reference electrode, and working electrode, respectively. A constant potential of $0.75 \mathrm{~V}$ was applied to the system for $600 \mathrm{~s}$, the obtained PPy film was removed from the electrolyte and rinsed with ultrapure water after electrodeposition.

The morphology of the PDA and PDA-PPy films were analyzed using SU-70 scanning electron microscopy (SEM). Adhesion force between the films and $\mathrm{Ti}$ substrate was tested by the Scotch ${ }^{\mathrm{TM}}$ Magic $^{\mathrm{TM}}$ Tape 810 $(3 \mathrm{M})$. The electrochemical impedance spectra were measured in $3.5 \% \mathrm{NaCl}$ solution at $25{ }^{\circ} \mathrm{C}$ with a $\mathrm{CHI}$ $660 \mathrm{E}$ Electrochemical Workstation using $5 \mathrm{mV}(\mathrm{rms}) \mathrm{AC}$ sinusoid signal at a frequency range from $1000 \mathrm{kHz}$ to $0.01 \mathrm{~Hz}$. The Tafel polarisation curves were performed by potentiodynamic polarisation in $3.5 \% \mathrm{NaCl}$ solution with a rate of $0.333 \mathrm{mV} \mathrm{s}^{-1}$.

\section{Results and Discussion}

Fig. 1 illustrated the potentiostatic method deposition of PPy films from SDBS and Py monomer solution on

\footnotetext{
a Corresponding author: zhongz@,xmu.edu.cn; gedt@xmu.edu.cn
} 
untreated Ti substrate, PDA modified Ti substrate, and pretreated plus PDA modified Ti substrate, the obtained samples were denoted as Ti/PPy, Ti/PDA-PPy and SC2Ti/PDA-PPy, respectively. As shown in Fig. 1, the current of all the electropolymerization curves initially decreases at the beginning and then increases at a fast rate, which correspond to $\mathrm{Ti}$ surface oxidation and PPy nucleation and growth, respectively[9]. The electropolymerization current of Ti/PPy was apparently larger than Ti/PDA-PPy and SC2Ti/PDA-PPy in the whole process, we deduced the current change was due to the pretreated process and the modification of PDA, which changed the surface state and electrical conductivity of Ti electrode, leading to the PPy formation more difficult.

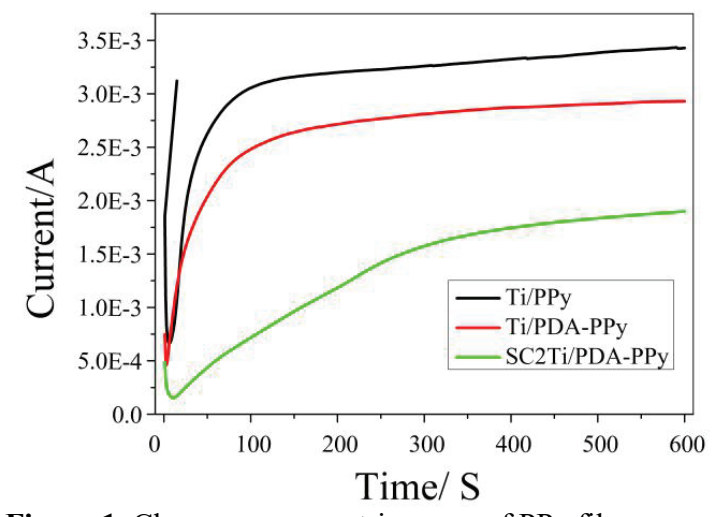

Figure 1. Chronoamperometric curve of PPy films

The morphology of the PDA and PPy films were characterized by SEM and shown in Fig. 2. From Fig. 2a and Fig. 2c, we can see that after PDA modification, lots of isolated islands were formed and the polishing scratch of $\mathrm{Ti}$ surface was coated by uniform thin film. It was consistent with the PDA layer formation process reported by Jiang et al[10], nanoaggregates of PDA and uniform PDA film were formed in the suspension and substrate simultaneously, and the particle of PDA will deposit onto substrate after a period of time. Fig. 2b and Fig. 2d shows the cauliflower-like morphology of PPy film, which
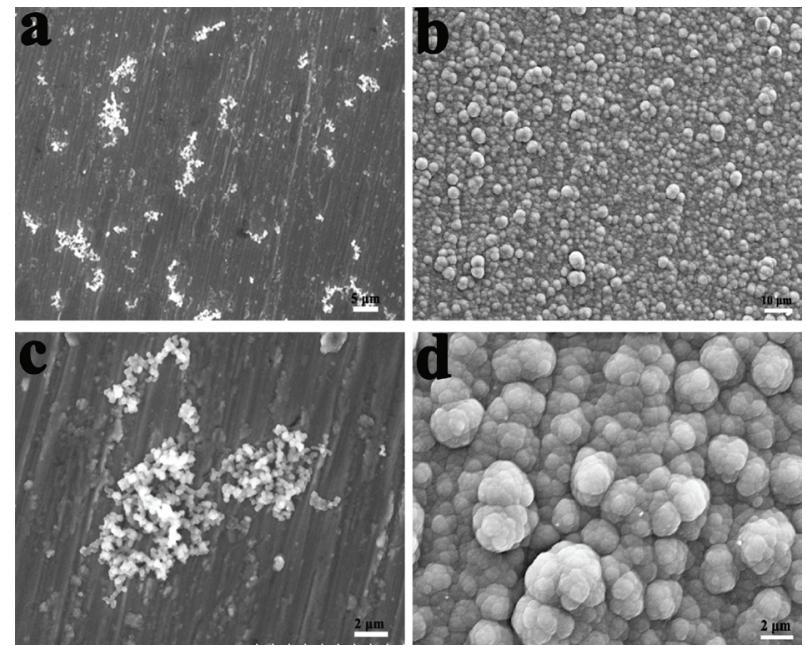

Figure 2. SEM images of PDA and PPy films. a) and b) was the low-resolution SEM images of PDA and PPy. c) and d) was the high-resolution SEM images of PDA and PPy. means the PDA modification or chemically pretreating for Ti substrate did not change the morphology of PPy films.

Adhesion force of the PPy and PDA-PPy films was examined by peel-off test after the electrosythesis, the results were shown in Table 1. For the Scotch tape peeloff test, a piece of tape was firstly taped on PPy films, the air bubbles was removed by pressure to make sure good contact between the tape and the film, then the tape was peeled off from the bottom upwards at a quick speed. If the film was strongly bonded with the substrate, the delamination will occur at the tape/film interface, otherwise the delamination will occur at the film/substrate interface. In the cases of PPy on untreated Ti, PPy films were completely removed from Ti surface for all 6 samples, indicating that PPy films were barely bonded to the substrate. In the cases of PPy on PDA modified Ti substrate, $20 \%$ of PPy films was removed from the Ti surface for 2 samples and $100 \%$ adhesion for other 4 samples, indicating that the cohesive strength between PPy film and Ti substrate was greatly enhanced. DA was a small biomolecule bearing similar structure to the essential adhesive component of mussel protein, so we supposed that the existence of PDA improved its adhesive force to $\mathrm{Ti}$ substrate. The best adhesive force between PPy and Ti substrate was in the cases of PPy on pretreated plus PDA modified Ti, 100\% adhesion was observed in all 6 samples, it was impossible to remove any PPy from the Ti substrate. We attribute the good adhesion to the pretreated process and the PDA modification, which change the topography and chemical composition of $\mathrm{Ti}$ substrate, leading to a more adhesive PPy film formation.

Table 1

Scotch tape test of PPy, and PDA-PPy films after the electrosythesis.

\begin{tabular}{lcc}
\hline \multicolumn{1}{c}{ Sample } & Number of samples & Result \\
\hline $\begin{array}{l}\text { PPy on Ti } \\
\text { (without any treatment) }\end{array}$ & 6 & $\begin{array}{l}100 \% \text { film off } \\
(6 \text { cases })\end{array}$ \\
$\begin{array}{l}\text { PDA-PPy on Ti } \\
\text { (without any treatment) }\end{array}$ & 6 & $\begin{array}{c}0 \% \text { film off ( } 4 \text { cases }) \\
20 \% \text { film off }(2 \text { cases })\end{array}$ \\
PDA-PPy on SC2 treated Ti & 6 & $0 \%$ film off ( 6 cases $)$
\end{tabular}

The corrosion resistance of PPy and PDA-PPy coated $\mathrm{Ti}$ in $3.5 \% \mathrm{NaCl}$ solution was analyzed by the open circuit potential, alternating current impedance test and tafel polarization test, the results was shown in Fig. 3. The open circuit potential (OCP, Fig. 3a) for the PPy coated Ti was positive shift to above than $0 \mathrm{~V}$ compared to pure Ti $(-0.33 \mathrm{~V})$, and the OCP of SC2Ti/PDA-PPy was more positive than Ti/PDA-PPy and Ti/PPy. Typical Nyquist plot of pure Ti, Ti/PPy, Ti/PDA-PPy, and SC2Ti/PDA-PPy was shown in Fig. 3b, all the curves showed only one capacitive loop in the measured frequency region. The enhancement of corrosion resistance can be reflected by the increasing radius of 
Nyquist plot[11]. It was observed that the radius of Ti/PPy was much higher than pure Ti, indicating the anticorrosion performance of $\mathrm{Ti} / \mathrm{PPy}$. The radius of SC2Ti/PDA-PPy was higher than Ti/PPy and Ti/PDA$\mathrm{PPy}$, indicating the best corrosion resistance. The tafel polarization test (Fig. 3c) further confirmed the enhanced corrosion resistance of Ti/PDA-PPy and SC2Ti/PDA-PPy. The positive shift in corrosion potential of Ti/PPy, Ti/PDA-PPy and SC2Ti/PDA-PPy (compared to pure Ti) was $0.289 \mathrm{~V}, 0.355 \mathrm{~V}$, and $0.372 \mathrm{~V}$, respectively.
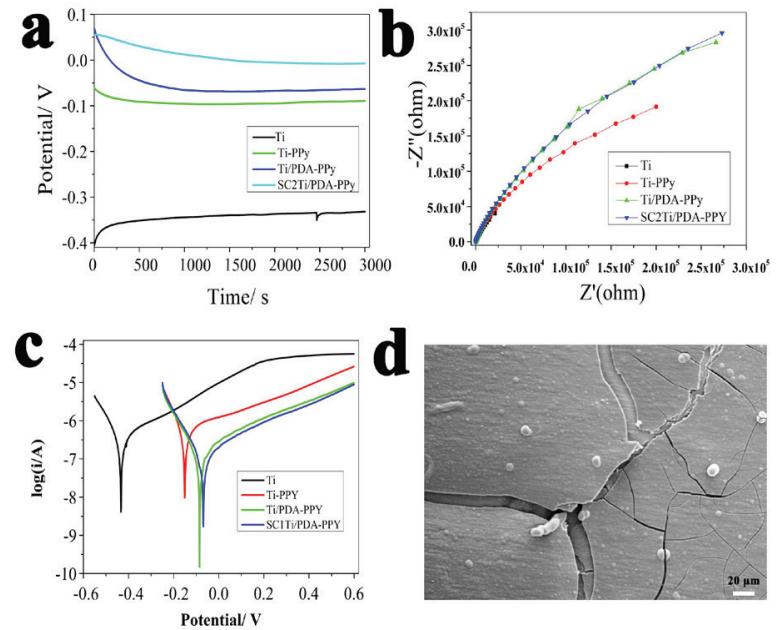

$\mathbf{e}$

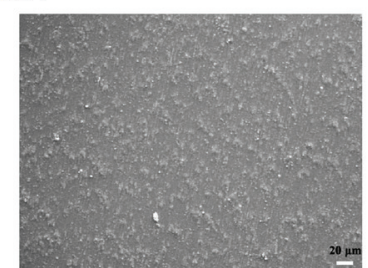

Figure 3. a) The OCP vs. time plot. b) The Nyquist plot. c) The tafel polarization plot of $\mathrm{Ti}$ and $\mathrm{PPy}$ coated $\mathrm{Ti}$ in $3.5 \% \mathrm{NaCl}$ solution. The SEM images of d) Ti/PPy and e) SC2Ti/PDA-PPy after polarization.

The corrosion rate for $\mathrm{Ti}, \mathrm{Ti} / \mathrm{PPy}, \mathrm{Ti} / \mathrm{PDA}-\mathrm{PPy}$, and SC2Ti/PDA-PPy was $4.29 \times 10^{-7} \mathrm{~m} / \mathrm{s}, 3.18 \times 10^{-7} \mathrm{~m} / \mathrm{s}$, $1.41 \times 10^{-7} \mathrm{~m} / \mathrm{s}$, and $1.34 \times 10^{-7} \mathrm{~m} / \mathrm{s}$, respectively. The much more positive shift in corrosion potential and the lower corrosion rate indicate the greatly enhanced anticorrosion performance of SC2Ti/PDA-PPy. The SEM images of Ti/PPy and SC2Ti/PDA-PPy after polarization was shown in Fig. 3d and Fig. 3e. It was observed that Ti/PPy film was badly damaged while the SC2Ti/PDAPPy film surface seems no change, which further verified the enhanced anti-corrosion performance of SC2Ti/PDAPPy.

\section{Conclusions}

Adherent SC2Ti/PDA-PPy film was electrosynthesized on chemical pretreat and PDA modified Ti substrate, the adhesion force between PPy film and substrate was evaluated by peel-off test. The adherent SC2Ti/PDA-PPy film showed improved protective behavior against the corrosion of $\mathrm{Ti}$ compared to pure $\mathrm{Ti}$ and $\mathrm{Ti} / \mathrm{PPy}$, which was reflected by open circuit potential, the polarization curves and the electrochemical impedance spectroscopy test. The results were also in agreement with the observed morphology of Ti/PPy film and SC2Ti/PDA-PPy film after polarization in $3.5 \% \mathrm{NaCl}$ solution.

\section{Acknowledgements}

This work was supported by the National Nature Science Foundation of China (31271009, 81271689), the Fundamental Research Funds for the Central Universities (No. 2011121001), the Program for New Century Excellent Talents in University, and the Program for New Century Excellent Talents in Fujian Province University and the Xiamen Municipal Science and Technology project (3502Z20144026).

\section{References}

1. N. Sheng, Y. Lei, A. Hyonoo, M. Ueda, T. Ohtsuka, Prog. Org. Coat 77, 1724 (2014).

2. M. Torabi, S.K. Sadrnezhaad, Mater. Corros 62, 252 (2011).

3. A.J.A. F. Faverolle, B. Bioch, P. Audebert, C. P. Andrieux, Chem. Mater 10, 13 (1998).

4. O.I. Katrin Idla, Marek Strandberg, Electrochim. Acta 45, 10 (2000).

5. S. Popescu, C. Ungureanu, A.M. Albu, C. Pirvu, Prog. Org. Coat 77, 1890 (2014).

6. H. Lee, S.M. Dellatore, W.M. Miller, P.B. Messersmith, Science 318, 426 (2007).

7. Q. Ye, F. Zhou, W. Liu, Chem. Soc. Rev 40, 4244 (2011).

8. Y. Liu, K. Ai, L. Lu, CHEM REV 114, 5057 (2014).

9. K. Cysewska, J. Karczewski, P. Jasiński, Mater. Lett 183, 397 (2016).

10. J. Jiang, L. Zhu, L. Zhu, B. Zhu, Y. Xu, Langmuir 27, 14180 (2011).

11. Z. Zhao, Y.W. Shao, T.M. Wang, D.P. Feng, W.M. Liu, Corros. Eng. Sci. Techn 46, 330 (2013). 